\title{
Preliminary results of ON/OFF detection using an integrated system for Parkinson's disease monitoring.
}

\author{
Matteo Pastorino, Jorge Cancela, Maria T. Arredondo, Laura Pastor-Sanz, Sara Contardi and Franco \\ Valzania
}

\begin{abstract}
This paper describes the experimental set up of a system composed by a set of wearable sensors devices for the recording of the motion signals and software algorithms for the signal analysis. This system is able to automatically detect and assess the severity of bradykinesia, tremor, dyskinesia and akinesia motor symptoms. Based on the assessment of the akinesia, the ON-OFF status of the patient is determined for each moment. The assessment performed through the automatic evaluation of the akinesia is compared with the status reported by the patients in their diaries. Preliminary results with a total recording period of $\mathbf{3 2}$ hours with two $\mathrm{PD}$ patients are presented, where a good correspondence $(88.2+/-3.7 \%)$ was observed. Best $(93.7 \%)$ and worst $(87 \%)$ correlation results are illustrated, together with the analysis of the automatic assessment of the akinesia symptom leading to the status determination. The results obtained are promising, and if confirmed with further data, this automatic assessment of PD motor symptoms will lead to a better adjustment of medication dosages and timing, cost savings and an improved quality of life of the patients.
\end{abstract}

\section{INTRODUCTION}

$\mathrm{P}_{\mathrm{n} \text { ens }}^{\mathrm{A}}$ ARKINSON'S disease (PD) is a progressive neurodegenerative disorder associated with motor dysfunction characterized by bradykinesia, resting tremor, muscle rigidity, and postural instability [1].

Current therapies are effective in managing Parkinsonian symptoms in the early stages of the disease. However, in late stage PD, patients develop motor complications, such as the abrupt loss of the efficacy of medications at the end of a dosing interval, involuntary hyperkinetic movements referred to as dyskinesia, gait dysfunctions such as festination, start hesitation and freezing of gait [2]. These impairments result in a highest propensity to falls, as well as a reduced capacity to walk [3], and lead to progressive functional restraints. Motor fluctuations during the day are also affected by medication timing, other drugs not related to PD, meals or sleeping deficiency [4].
Currently, motor assessment in PD is mainly based on historical information, home diaries and neurological examination during visits to the clinic. These methods clearly suffer from many limitations: data can be highly subjective, they depend on patient's memory that could be affected by the disease, patients could unconsciously exaggerate or attenuate symptoms' severity, and there could be a lack of comprehension because of a poor capacity to understand medical terminology or insufficient experience of the physician in the field. Moreover, most of the patients may not be aware of mild tremor or dyskinesia.

In an attempt to solve these problems and to find more objective assessments, several scales, based on impairment [5][6][7], disability [8][9] and quality of life [10], have been designed and used, but all them suffer from limits: intra and inter-observer inconsistencies and subjectivity issues related to historical information. Moreover, the pattern and severity of PD symptoms may vary considerably during the day, while clinical rating scales only provide moment-to-moment assessments; and finally, measurements of motor functions made in the clinic may not accurately reflect the actual functional disability experienced by the patients in their daily life. In addition to rating scales, akinesia tremor, rigidity and gait and postural stability are sometimes evaluated by means of motor performance test, but they are limited to the setting of the clinic or laboratory. Direct observation by a PD specialist, as an alternative to historical information revision, results impractical because motor fluctuations cover the time of several hours between medication dosages.

This paper describes an integrated platform to enable access to data gathered using wearable sensors via a web application. PERFORM project aims at testing an innovative and reliable tool, for monitoring PD patients at home. PERFORM wearable system could objectively monitor and quantify disease fluctuation across the day, in function of meals, drugs and sleep. The clinician can remotely monitor the overall status of the patients, adjust medication schedules and personalize treatment. Collecting data in the home setting using this system has the potential to allow clinicians to improve quality of care in patients with $\mathrm{PD}$, also reducing its costs.

\section{STUDY DESCRIPTION}

\section{A. System for data collection}

An integrated system, composed by a set of wearable sensors devices for the recording of the motion signals and software 
algorithms for the signal analysis, has been used in order to constantly acquire motor signals of the patients.

The hardware is formed by a set of four tri-axial accelerometers positioned at each patient limb used to record signals from legs and harms; a belt sensor, composed by an accelerometer and a gyroscope, used to record body movement accelerations and angular rate; and a data logger used to receive and store all recorded signals in a SD card. All sensors transmit data using Zigbee protocol to the logger device, with $62.5 \mathrm{~Hz}$ sampling rate.

Data acquired are pre-processed by advanced knowledge processing methods, integrated by fusion algorithms to allow health professionals to remotely monitor the overall status of the patients, adjust medication schedules and personalize treatment. Adjustment of treatment occurs through the capability of keeping track of the timing and doses of the medication and meals that the patient is taking [12].

\section{B. Experimental Setup}

The objective of the exposed experimental is assessing the motor status of patients with Parkinson's disease in an unsupervised environment, with the aim of obtaining an objective assessment of motor performance in relation to the consumption of drugs.

In order to achieve this goal, the patients were asked to wear the sensors at home during a week. The schedule of the recording sessions was programmed by a technician directly at the patient's house, discussing with him/her the most appropriate timing. The patient was requested to wear the devices 10 minutes before the start of the pre-scheduled monitoring session, then he/she was free to do his/her everyday activities. During the day the patient had to introduce the information about the medication and meal intake, using the developed User Interface. At the end of the day the patient had to connect the data logger to the $\mathrm{PC}$, where the data downloading and signal processing started automatically.

\section{Patients data}

Patients fulfilling the following criteria were eligible for the study: diagnosis of Parkinson's disease, aged between 40-75 years old, ambulatory and capable of complying with study requirements, receiving stable dopaminergic treatment, experiencing motor fluctuations and presence of a responsible caregiver who could cooperate with the patient and his/her neurology specialist. The exclusion criteria for this study were suffering from dementia, psychosis (simple visual hallucinations excluded) or a significant systemic disease (such as: cancer, hepatic or kidney dysfunction, etc.).

During the trials, 25 PD patients were selected ( 8 women and 17 men) with an age ranging from 41 and 79 years old (mean $60.9 \pm 9.0$ years)

\section{RESULtS}

The aim of the experiment was to test the behavior of the developed system in an uncontrolled environment. In order to evaluate and validate the general architecture, results of the automatic assessment are compared with the results of the patient diary. A proof of concept of the system with 2 patients, including the best and the worst case is presented. Furthermore, the result of the compliance and level of comfort evaluation of the prototype system is illustrated, including the results of the $25 \mathrm{PD}$ patients tested in Modena, Italy.

\section{A. ON/OFF Evaluation. Methodology and assessment.}

During the trial phase all patients filled a diary during two consecutive days. Patients were asked to indicate their motor condition every half an hour during the day, using a 3 level classification (OFF, ON without dyskinesia and $\mathrm{ON}$ with dyskinesia).

It was then performed a correlation analysis of the daily diary classification with the output of the system ON/OFF evaluation based on the results of the classifiers developed for the recognition of each symptom. The aim was to evaluate the capability of system to automatically discriminate ON/OFF conditions.

This paper present the results of the test carried out for two different patients during the last phase of PERFORM project trials in Modena, Italy. Considering the time frame during which the data were comparable to patient diary (2 daily sessions of 4 hours for 2 patients during 2 days, i.e. total of 32 hours) a good correspondence $(88.2+/-3.7 \%$ ) between patient's diary and ON/OFF phases identified by the system was observed. Hereinafter the best and the worst case are presented, together with the explanation of the results compared for the particular case of the automatic assessment of the Akinesia [12].

Fig. 1 shows the best case results. The black line describes the general ON/OFF trend as result of the information introduced by patient in the diary. The gray line indicates the ON/OFF evaluation based on the automatic assessment of the PD symptom by the system under evaluation. The correlation between the patient's diary and the automatic assessment reaches $93.7 \%$.

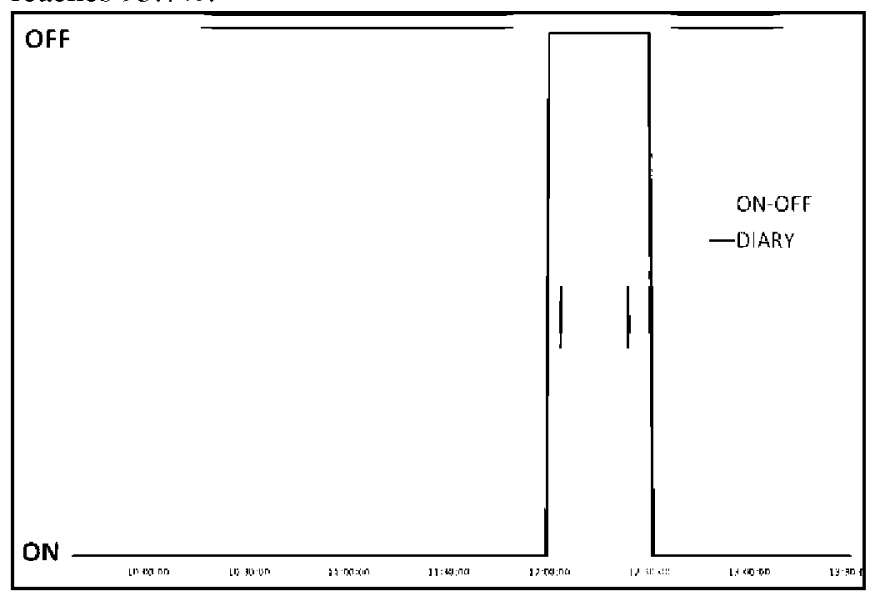

Fig. 1 ON/OFF results comparison of the diary and the overall status assessment using the automatic assessment of the PERFORM system - Best Case.

A small delay around the transition phases ON/OFF (Fig. 1) is observed. In fact, since the diaries were filled every 30 
minutes, they produced a relevant degree of data roughness. On the other hand, the system for the automatic assessment registered real-time signals, allowing an exact description of the motor status.

From a therapeutic point of view even a delay of 10-15 minutes, though seemingly short, may be crucial in patients with motor fluctuation. In fact, it is known that taking a new LDopa medication when a previous dose effect has not been finished (still in ON condition) cause a more stable and a good global response with lower dosage of the drug. Moreover, if Ldopa is taken when the effect of the previous dose has completely extinguished (OFF-condition) the clinical response is unpredictable (absent, delayed and intermittent). Therefore, having an accurate ON/OFF indicator system can help clinicians to plan a more effective therapeutic schedule.

One of the most characteristic PD symptoms is the Akinesia [12], that describes the general daily evolution of the syndrome, computing the energy produced along the day. Fig. 2 shows the evolution of akinesia corresponding to the result of the comparison showed in Fig. 1. In this case the $\mathrm{ON}$ and OFF transition can be easily detected using the output of the automatic assessment of the system thanks to the data collected in both phases.

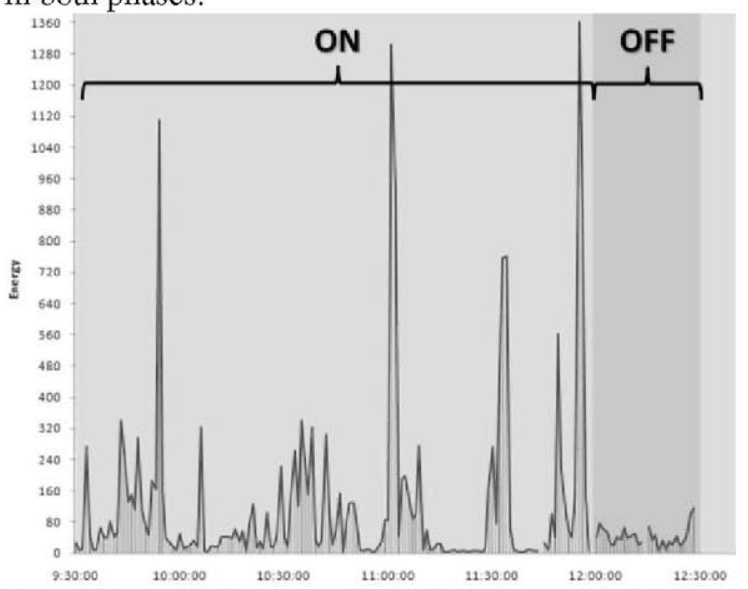

Fig. 2 Akinesia level corresponding to the best case of ON/OFF comparison.

Fig. 3 shows the worst case results. The correlation between the patient's diary and the automatic assessment reaches $87 \%$, but the OFF phase detected by the automatic assessment of the PD symptom does not match the OFF phase indicated by the patient.

This mismatching can be caused by different reasons. Analysing Fig. 4 the patient, during the second part of the recording, has pointed as $\mathrm{OFF}$ and $\mathrm{ON}$ two conditions not particularly different from each other. Considering the analyses based on the quantitative data obtained from the PERFORM system, it is evident that these two conditions are more likely a partial OFF status followed by a partial ON status.

The advantage of a better definition of the motor status results in a more appropriate adjustment of the drug dosage. Moreover, during the OFF phase indicate by the patients, a block, and a corresponding lack of data, is detected with the analysis of akinesia, therefore the algorithms cannot work properly.

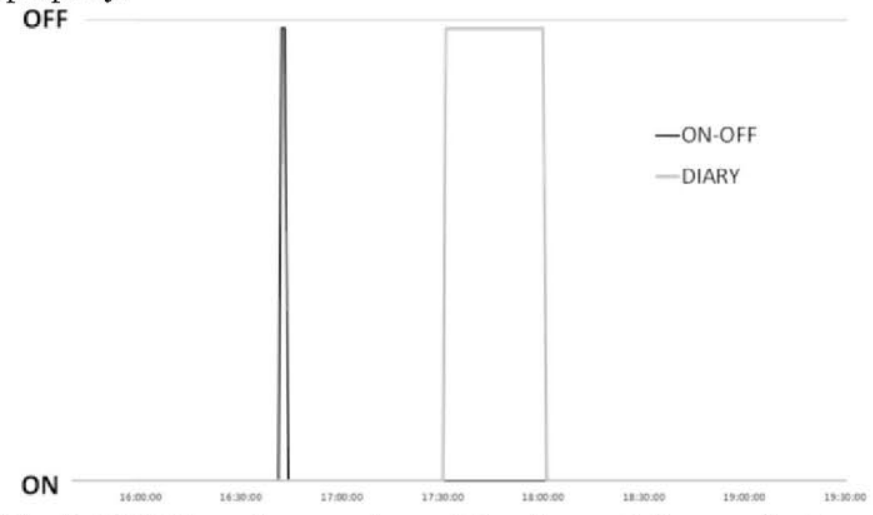

Fig. 3 ON/OFF results comparison of the diary and the overall status assessment using the automatic assessment of the PERFORM system - Worst Case.

In particular, in this specific patient, in view of the system assessment, it was decided to increase by $25 \mathrm{mgs}$ (from 100 to 125) the L-dopa dose taken at $5 \mathrm{pm}$. As a result, in the next week patient reported a disappearance of that $\mathrm{OFF}$ phase.

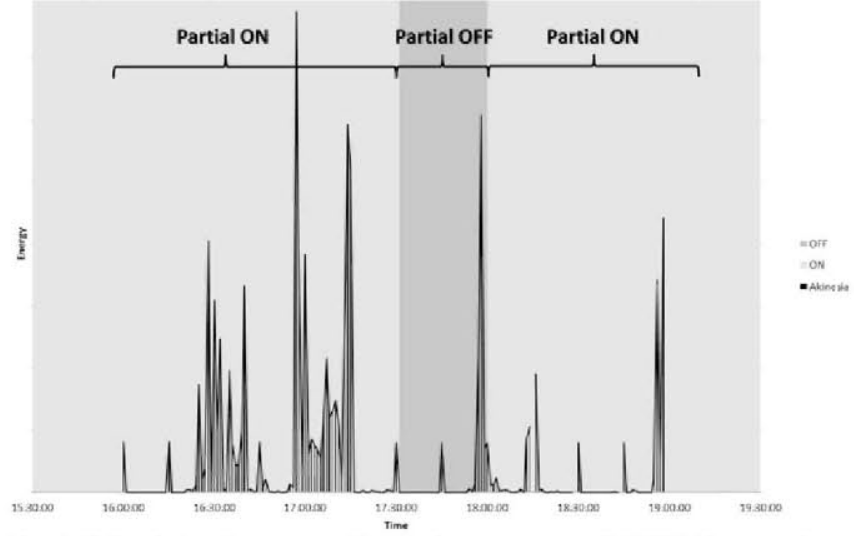

Fig. 4 Akinesia level corresponding to the worst case of ON/OFF comparison

Furthermore, the analysis of the quantitative data provided correlations with activities indicated by patient (walk, rest, hand-activities) even related with the specific patient job. In this case the subject is a dentist and he reported, at the end of the morning, difficulties in performing a tooth extraction which caused embarrassment in front of his patient.

\section{B. Compliance and level of comfort}

An evaluation regarding the wearability of the system was performed at the end of every trial session, in order to produce a global evaluation about level of comfort. All the evaluations were based on a scale composed of five items, with different evaluation levels (poor, insufficient, sufficient, good and optimal).

Generally users did not face any problem using the system, placing the overall compliance level between good and optimal. As showed in Fig. 5, 16 of 25 subjects (65\%) reported that the system was comfortable (good/optimal) and that it could be used for monitoring clinical state outside of experimental conditions. Four patients reported that the system was not comfortable mainly due to the dimension and weight 
of the wearable devices and the frequent loss of connection.

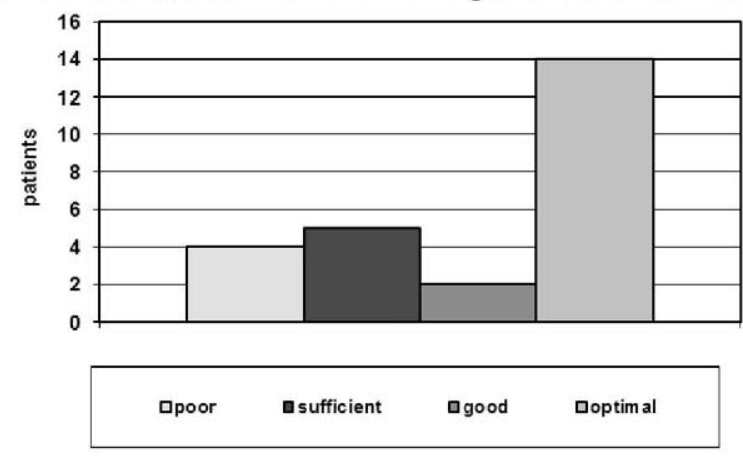

Fig. 5 Comfort evaluation results obtained in the last trials phase.

\section{CONCluSion}

An innovative wearable system for the remote monitoring and evaluation of PD motor symptoms has been described. The results of the assessment performed by a specialist have been compared with diaries and system data for two patients. They have been presented as proof of concept of the system clinical value for the discrimination of $\mathrm{ON}$ and OFF states. In addition, an evaluation regarding the comfort of the system has been performed with 25 users, suggesting that it is generally regarded as usable.

\section{A. Impact}

Better control of the disease symptoms will have a synergistic positive effect on the psychosocial and physical functioning of the patients. Therefore patients are empowered to play a full role in society by living independently, working, participating in social activities and enjoying life.

\section{B. Medical and Innovation utility}

This system is expected to provide work efficiency to healthcare professionals (e.g. through the utilisation of multiparametric information fusion and consistent decision support tools, remote monitoring, reduction of patient visits health assessment and treatment adjustment). In addition, it will allow the advance in medical research through the exploitation of the vast pool of monitored parameters and the generated statistical data for the production of new diagnostic models and protocols. Although the application domain is the Parkinson's disease, there seems to be a great potential and easy future exploitation in other target groups such as other neurodegenerative diseases with similar motor and mental disabilities.

\section{Low Cost System for chronicle disease}

This system will achieve cost and effort savings related to frequent visits to hospitals for assessment and treatment modification, considering that most PD patients are elderly, present mobility impairments and may live in rural areas. In addition, an optimal diagnosis and monitoring is expected to decrease the expenses in drugs, and therefore reduce the costs associated to the disease follow up.

The results presented in this paper are promising, even if more work will be done in order to give a complete clinical validation. In particular, future work will be focused on the improvement of the wearable system, including mobile technology, making it more portable and usable. Moreover, future improvement will be dealing with the "lack of data" period. For example, a mobile application associated to the system, will be able to request to the patient about the actual $\mathrm{ON}-\mathrm{OFF}$ state during rest periods.

\section{ACKNOWLEDGMENT}

Authors thank the PERFORM consortium for their contribution to this work, especially the University Clinic of Navarra (Spain), the University of Ioannina (Greece), the Nuovo Ospedale Civile di Modena (Italy), Dr. Laura Rocchi of the University of Bologna (Italy) and Professors José Antonio Martín Pereda, Ana González Marcos, Paloma Rodríguez Horche and Francisco del Pozo of the Technical University of Madrid (UPM - Spain).

\section{REFERENCES}

[1] J. Jankovic, "Parkinson's disease: clinical features and diagnosis," Journal of Neurology Neurosurgery and Psychiatry, vol. 79, no. 4, pp. 368-376, 2008.

[2] T. A. Boonstra, H. Van Der Kooij, M. Munneke, and B. R. Bloem, "Gait disorders and balance disturbances in Parkinson's disease: clinical update and pathophysiology.," Current Opinion in Neurology, vol. 21, no. 4, pp. 461-471, 2008.

[3] D. Tan, M. Danoudis, J. McGinley, and M. E. Morris, "Relationships between motor aspects of gait impairments and activity limitations in people with Parkinson's disease: a systematic review.," Parkinsonism \& related disorders, vol. 18, no. 2, pp. 117-24, Feb. 2012.

[4] S. Fahn, "Medical treatment of Parkinson's disease," Journal of Neurology, vol. 245, no. 3, p. P15-P24, Sep. 1998.

[5] N. Giladi et al., "Validation of the freezing of gait questionnaire in patients with Parkinson's disease.," Movement disorders: official journal of the Movement Disorder Society, vol. 24, no. 5, pp. 655-61, Apr. 2009.

[6] G. Abbruzzese et al., "Linguistic, psychometric validation and diagnostic ability assessment of an Italian version of a 19-item wearingoff questionnaire for wearing-off detection in Parkinson's disease.," Neurological sciences: official journal of the Italian Neurological Society and of the Italian Society of Clinical Neurophysiology, pp. 1-9, Feb. 2012.

[7] P. Martinez-Martin et al., "International study on the psychometric attributes of the non-motor symptoms scale in Parkinson disease.," Neurology, vol. 73, no. 19, pp. 1584-1591, 2009.

[8] M. M. Hoehn and M. D. Yahr, "Parkinsonism: onset, progression and mortality," Neurology, vol. 17, no. 2, pp. 318 and 16 pages following, 1967.

[9] P. Martinez-Martin et al., 'Unified Parkinson's disease rating scale characteristics and structure," Movement Disorders, vol. 9, no. 1, pp. 76-83, 1994.

[10] V. Peto, C. Jenkinson, and R. Fitzpatrick, "PDQ-39: a review of the development, validation and application of a Parkinson's disease quality of life questionnaire and its associated measures," Journal of Neurology, vol. 245, no. 1, p. S10-S14, Apr. 1998.

[11] L. Pastor-Sanz, M. Pansera, J. Cancela, M. Pastorino, and M. T. Arredondo Waldmeyer, "Mobile Systems as a Challenge for Neurological Diseases Management - The Case of Parkinson's Disease," in Diagnostics and Rehabilitation of Parkinson's Disease, J. Dushanova, Ed. InTech, 2011, pp. 287-308.

[12] A. Pascual-Leone, J. Valls-Sole, J. P. Brasil-Neto, L. G. Cohen, and M. Hallett, "Akinesia in Parkinson's disease. I. Shortening of simple reaction time with focal, single-pulse transcranial magnetic stimulation.," 1994. 